\title{
Seventh User Workshop on High-Power Lasers at the Linac Coherent Light Source
}

\author{
Gilliss Dyer ${ }^{1, *(D)}$, Cindy Bolme ${ }^{2}\left(\mathbb{D}\right.$ and Siegfried Glenzer ${ }^{1}[$ \\ 1 SLAC National Accelerator Laboratory, Menlo Park, CA 94025, USA; glenzer@slac.stanford.edu \\ 2 Los Alamos National Laboratory, Los Alamos, NM 87544, USA; cbolme@lanl.gov \\ * Correspondence: gilliss@slac.stanford.edu
}

Received: 28 April 2020; Accepted: 29 April 2020; Published: 16 May 2020

\begin{abstract}
We report on a seventh annual workshop in a series focused on science realized by the combination of hard X-ray free electron lasers with high power optical lasers, hosted at the SLAC National Accelerator Laboratory in Menlo Park, CA. Members from the user community of the Matter in Extreme Conditions (MEC) endstation of the Linac Coherent Light Source (LCLS) and other scientists met with local scientists to discuss developments at LCLS and MEC and related facilities, including experimental results and future plans.
\end{abstract}

Keywords: X-ray free electron lasers; high energy density; ultrafast lasers; laser matter interaction

\section{Introduction}

On September 26th and 27th in Menlo Park, California, SLAC National Accelerator Laboratory (SLAC) hosted the international user workshop on science realized by the combination of the Linac Coherent Light Source (LCLS) with high-power lasers. This was the seventh annual workshop in a series centered around the science and developments of the Matter in Extreme Conditions instrument (MEC) at LCLS and touching also on similar facilities being developed worldwide, as well as related science at SLAC [1-6]. In the year since the last workshop, 11 journal articles [7-17], and one comment [18] have been published on research at LCLS-MEC. This year's workshop was co-organized by Los Alamos National Laboratory and SLAC National Accelerator Laboratory. There were 79 registered participants.

For the third year, the workshop overlapped with the SSRL/LCLS User Meeting, and plenary sessions from that meeting were incorporated into the morning of the workshop's first day. These included a welcome message from SLAC director Chi-Chang Kao, a Department of Energy (DOE)-Basic Energy Sciences (BES) update from BES Director of Scientific User Facilities Jim Murphy, and LCLS and SSRL updates by LCLS and SSRL Directors Mike Dunne and Paul McIntyre, followed by a town hall discussion with the directors. In his talk, Dunne reflected on the 10 year anniversary of LCLS operations and discussed exciting future projects, including the LCLS-II upgrade [19] being installed during the 2019 shutdown, the LCLS-II-HE extension, new LDRD initiatives such as concepts to achieve XFEL temporal coherence [20], and the MEC Upgrade Project. The high power laser workshop continued with independent sessions in the afternoon and through the following day, covering major upgrade plans for MEC, other MEC-relevant developments at LCLS, updates on the High Energy Density (HED) endstations at SACLA and European XFEL, and a review of experiments from the abbreviated Run 17 of LCLS.

This year has brought major changes at LCLS, with the facility unavailable for all of calendar year 2019 due to the upgrade to LCLS-II. This upgrade project is 90\% complete, and the X-ray Free Electron Laser (XFEL) is expected to resume user operations with the $\mathrm{Cu}$ linac in 2020. The new superconducting linac is expected to be available for users experiments in late 2021. The upcoming Run 18 (the schedule for which is currently being reworked in light of the Covid-19 pandemic) will feature new variable 
gap undulators and a new suite of X-ray detectors (ePix10k, Rayonix 3.6 MPixel, and Jungfrau 4M). LCLS introduced a new proposal mode for Run 18: Science campaigns will provide beam time allocation for a series of experiments over several run cycles. These campaigns will be targeted on addressing high-impact scientific goals, and aim to close the loop on the make-measure-model cycle. In addition to the experimental upgrades, transformations are also underway with the LCLS data systems. Data scientists are developing targeted workflows for data detection, reduction, real-time analysis, and interpretation. These efforts will produce modular data workflows to expedite data processing. LCLS has recently published a long-term facility strategy will additional details, cf. LCLS Strategic Facility Development Plan [21].

\section{The MEC Upgrade}

Following a tour of MEC and opening remarks by MEC Department Head Gilliss Dyer, Project Director Alan Fry presented the status of the design for the MEC upgrade project at LCLS. In 2019, FES issued a mission need statement, initiating a formal $413.3 \mathrm{~b}$ project at SLAC to develop a conceptual design. Fry reminded participants that serious discussion of petawatt upgrades to MEC had started in 2014, including during the Second HPL Workshop [2]. The idea had gained momentum in 2018, with increasing calls for the U.S. to regain a position of world leadership in high power lasers, such as in the National Academies of Science, Engineering and Medicine (NASEM) report "Opportunities in Intense Ultrafast Lasers: Reaching for The Brightest Light" [22]. Fry emphasized that the conceptual design of the MEC upgrade has been strongly influenced by the Brightest Light Initiative (BLI) workshop [23], a high power laser community response to the NASEM report. This includes a push for higher repetition rates in high pulse energy, high peak power laser systems, both to improve feedback for better beam quality and increase potential data rates. Also, state-of-the-art yet currently available laser technology could be used in the MEC upgrade to create revolutionary capability with reduced risk relative to inventing new laser technologies.

This high intensity laser upgrade will require the addition of significant radiation shielding around the target areas [24]. SLAC has investigated options for adding a radiation shielding wall within the current Far Experimental Hall (FEH), but the disruption of constructing sufficient shielding within the FEH was deemed prohibitive. Instead, the plan calls for excavation of a new experimental hall beyond the $\mathrm{FEH}$, to house new experimental areas appropriate for combining petawatt- and kiloJoule- class lasers with the LCLS hard X-ray beam, while leaving space for future significant upgrades. The pre-conceptual layout for this new experimental hall at the time of the workshop is shown in Figure 1. A candidate for the petawatt laser would be a diode pumped glass laser producing pulses with over $100 \mathrm{~J}$ at up to $10 \mathrm{~Hz}$ and able to deliver nanosecond shaped pulses, while a $\mathrm{kJ}$ class nanosecond shock drive laser would fire at a rate similar to the current MEC $60 \mathrm{~J}$ system.

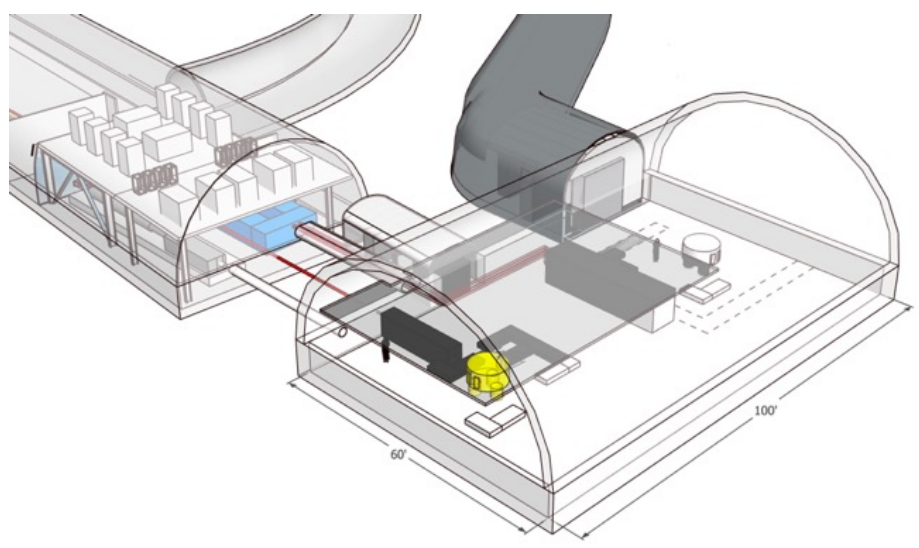

Figure 1. Pre-conceptual layout of new experimental hall for MEC laser upgrade project at the time of the workshop. The excavation shown is $60^{\prime} \times 100^{\prime}$ and would contain two target areas, one on the $\mathrm{X}$-ray line and one off. 
High Energy Density (HED) Division Director Siegfried Glenzer next presented an overview of HED science applications for bright hard X-ray lasers. Their unmatched probing capabilities make possible revelatory measurements of relativistic plasma instabilities, such as those relevant to cosmic ray formation, strongly coupled plasmas, ultrafast radiation damage cascades, and phase transitions of transient high pressure matter. Such precision measurements can be applied to validating the predictions of PIC simulations, Density Functional Theory, molecular dynamics (MD) modeling, and equations of state. He presented the opportunity to resolve dislocation dynamics, and validate MD simulations, as an example of a major advantage arising from the higher photon energies made available by the LCLS-II upgrade. Glenzer expressed support for the strategy of pursuing a PW laser of higher energy than the more common Ti:Sapphire architecture used at other XFELs, based on experiments suggesting much higher brightness ion source, necessary for single shot radiation damage studies, and simulations showing novel plasma physics states that could be driven by the laser. He showed ion acoustic data as an example of a class of experiment that will benefit from a high repetition rate, requiring the accumulation of small signals. He further supported the inclusion of a kJ laser system in the upgrade for an expansion of the range of high pressure phase space accessible to precision X-ray measurements. For this application especially, he encouraged development of detectors capable of reading out the unique multi-pulse mode being developed at LCLS, wherein the facility can deliver 4-8 pulses separated by multiples of $0.7 \mathrm{~ns}$ (two RF bucket separation), with the potential to reach $360 \mathrm{ps}$ [25]. This mode would enable multi-frame measurements of the progression of a single shocked system.

\section{MEC Status and Discussion}

\subsection{MEC Status Update}

MEC Department Head Gilliss Dyer opened the second day with an overview of recent developments at MEC, leading into the user discussion of needs for the MEC instrument, both in the near term and in the proposed upgrade.

Recent years' upgrades to the long pulse laser are resulting in better stability, performance, and control of the laser drive conditions. The pulse shaping and its algorithms are continuing to be refined, and the issues that led to spontaneous small glitches in pulse shape, occasionally seen during run cycle 17, have been resolved. A recent investment in additional continuous phase plates (CPP) has improved the homogeneity of the beam spatial profile for more beam sizes. These new phase plates were manufactured by LLNL, and they produce spot size diameters of 150, 300, and $600 \mu \mathrm{m}$.

Some on-shot technical issues with the long pulse laser persist. Shots using the long pulse laser are still frequently causing fratricide of adjacent targets on a target grid. High energy shots are continuing to produce electromagnetic pulses (EMP) that detrimentally affect the CSPAD quad detectors. Several discrete failure modes have been identified due to the EMP. Electrical isolation schemes have been only partly effective, and the investigation is on-going. New quad detectors based on the ePIX10k platform [26] are now being built by SLAC's Technology Innovation Directorate (TID) to replace the CSPADs. Aside from their many advantages in signal quality and higher sensitivity at high photon energy, the MEC quads are also being designed using best practices to protect against strong EMP. These changes make them slightly bulkier but, hopefully, much more robust against EMP.

The short pulse laser can operate in three modes: standard short pulse, frequency doubled short pulse, and uncompressed. For standard short pulse mode, the laser delivers $1 \mathrm{~J}$ in $40 \mathrm{fs}$ of $800 \mathrm{~nm}$ at $5 \mathrm{~Hz}$. The pulse cleaner upgrade discussed at the previous workshop [6] has continued to operate stably and deliver excellent pulse contrast; its design and performance are described in Applied Physics Letters [27]. Even better contrast can be achieved using frequency doubling, for which pulses up to $400 \mathrm{~mJ}$ have been delivered for an experiment in run 16 requiring maximum pulse contrast [28]. Additional short pulse laser modes using the uncompressed pulse and using the laser at $2 \omega$ have been used for recent experiments. Uncompressed mode bypasses the compressor to deliver 130 ps pulses 
with roughly $1.5 \mathrm{~J}$ of energy at $5 \mathrm{~Hz}$. A new standardized platform of beam delivery was defined for Run 18, based on an F/5 focus, and is shown in Figure 2.

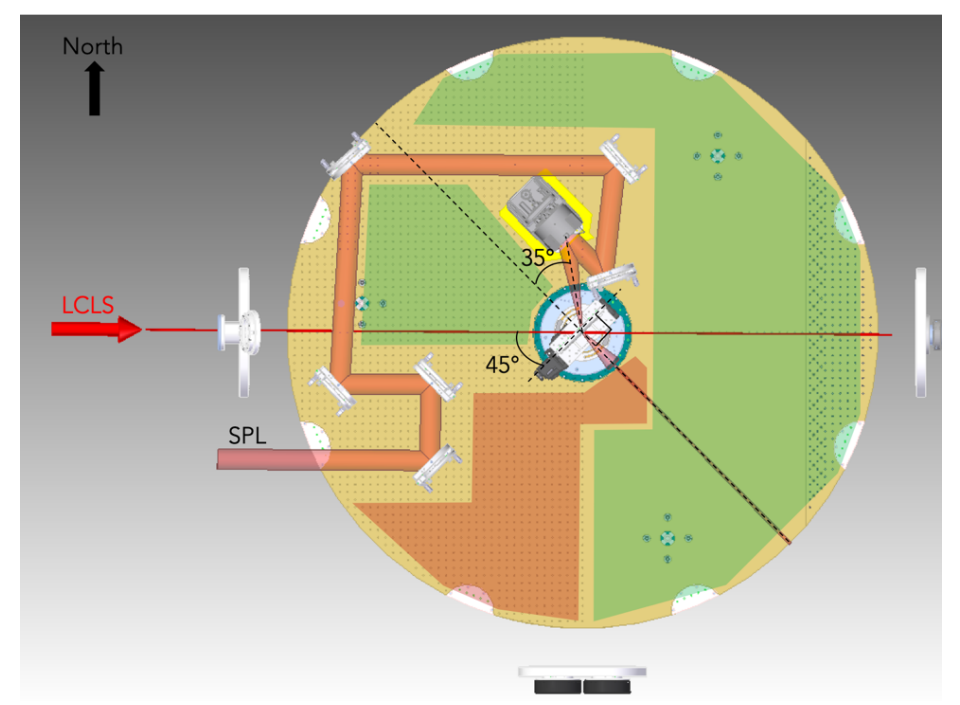

Figure 2. Top view of the standard short pulse laser delivery platform. Green indicates allowable space for user diagnostics; red is reserved for laser diagnostics

X-ray background from ultrashort pulse laser matter interaction can overwhelm faint scattering signals in MEC experiments, as noted in the talk by Sawada. This background, mostly bremsstrahlung, will become a more significant issue with higher power lasers, as further discussed in the talk by Zastrau. MEC has been studying more shielded spectrometer configurations and diagnosing the background levels in laser-only in-house experiments.

Current diagnostic developments include a new MEC X-ray Imaging and nano-focus platform for run cycle 18, a single shot laser energy measurement diagnostic, and a new highly annealed pyrolitic graphite (HAPG) spectrometer design covering the higher photon energies of LCLS-II.

The LCLS-II upgrade brings with it a new dedicated hard X-ray undulator (HXU) alongside a soft X-ray undulator to replace the single undulator of the original LCLS. This change brings several advantages for MEC. First, a higher photon energy of up to $24 \mathrm{keV}$ in the fundamental. This energy could be reached previously in the third harmonic, but at much lower pulse energy. Currently, mirror coatings restrict the photon energy to around $30 \mathrm{keV}$, but new mirror configurations and coatings are under consideration to access $40 \mathrm{keV}$ or higher in the third harmonic with the new HXU. The HXU is a variable-gap design, meaning that the $\mathrm{X}$-ray energy should be much faster to tune within the $\pm 10 \%$ range than previously. It is also worth noting that the polarization will change from horizontal to vertical. Finally, the availability of the dedicated HXU means that MEC is sharing time with only four instruments, and available $X$-ray time is expected to eventually grow by over $60 \%$.

MEC has expanded its operations outside of just LCLS-based experiments and at the time of the workshop was supporting the first experimental run of LaserNetUS at MEC.

\subsection{MEC Users Discussion}

MEC distributed a survey to all workshop participants to collect feedback regarding near-term desired improvements and long-term laser upgrade directions. The survey responses guided the user discussion session, which is summarized below.

\subsubsection{Current Laser Systems and Needs}

Long pulse laser: Suggested improvements to the long pulse laser include the addition of more diagnostics through the laser system and transitioning the output laser energy adjustment to after 
instead of before the $2 \omega$ crystals. Previous diagnostic improvements identified a slow drift in the phase matching angle in the doubling crystals, which could result in decreased output energy over the course of the day.

Short pulse laser: The pointing stability of the short pulse laser is currently one of the top user concerns. MEC has been working on replacing key mounts with high stability mounts, which has already significantly increased the stability. High stability motorized mounts have been purchased for transitions between the laser sections, and active stabilization of the beam pointing will be implemented. There was also discussion about the possibility of using the long pulse and the short pulse lasers concurrently. This experimental configuration is possible but has not yet been done. Users interested in this configuration should keep in mind that the long pulse laser currently has a jitter of $\sim 20$ ps with respect to LCLS and the short pulse laser.

\subsubsection{Priorities for Standard Configurations}

Long pulse laser: Users expressed that the standard configuration for X-ray diffraction needs to be modified for the higher energy X-rays that will be produced by LCLS-II. Detector coverage needs to shift to lower angles with users suggesting the range of $5-50^{\circ}$. An issue with shifting detectors to lower angles is that the VISAR lens currently obscures $\sim 15^{\circ}$. MEC committed to work with users in adjusting the standard configuration detector positions in the upcoming run cycle 18 and will explore options for having separate standard detector configurations for low X-ray photon energies and for high $X$-ray photon energies. The $X$-ray Thomson scattering spectrometers are undergoing improvements to support higher photon energies.

Short pulse laser: Laser delivery and characterization will be standardized for run 18 with $\mathrm{f} / 5$ focusing. Suggestions for additional standard configuration diagnostic platforms included small angle scattering and the combination of imaging and diffraction.

\subsubsection{Diagnostics}

XFEL: Users expressed desire for XFEL diagnostics to include the number of photons and the X-ray spectrum for each shot. Restoration of the upstream spectral measurement capability and implementation of a variable monochromator are highly desired, and these spectral capabilities might be able to be shared by multiple hutches if they are installed far enough upstream.

Long pulse laser: Desired diagnostics for the long pulse laser are laser spatial profiles on each shot. MEC is still investigating the best route to achieving this, as the phase plates are necessarily placed immediately before the main focusing lenses.

Short pulse laser: Users noted that a diagnostic of the intensity and spectrum of the petawatt-produced X-ray emission would be highly valuable for all short pulse laser experiments.

Timing: Recent changes to the VISAR system have improved the laser coupling efficiency into the fiber, and the streak cameras have been repaired. Addition of timing fiducials and a timing comb to the streak camera records is desired. The time tool in MEC is not a standard diagnostic, and more resources would be needed to improve its reliability.

\subsubsection{Future FEL Capabilities}

Users universally communicated that the highest XFEL priorities for MEC should include making use of the multi-bunch capability [25] and the ability to deliver narrower X-ray bandwidth. Narrow bandwidth pulses should have the best possible intensity stability and highest peak brightness. Users noted that several experiments were not submitted for run 18 because the availability of self-seeding was uncertain, and emphasized this as a high priority. Users also expressed interest in the possibilities for MEC science of cavity-based X-ray techniques [20] now being pursued via SLAC lab-directed research and development (LDRD). 


\subsubsection{Long-Term Upgrade Plans}

The user community was excited about the prospects for the major MEC upgrade project, and they stated the following highly desired features:

- Physical layout and radiation shielding for the two experimental areas that would allow experiment qualification in a laser-only chamber, as described in the pre-concept.

- Two chambers (one smaller and more specialized) to be installed in the target area situated on the LCLS beam path to facilitate rapid switching between experimental configurations, similar to the HED capability at European XFEL (Section 5.1).

- Options for X-ray focusing using KB mirrors, which have less chromatic dependence than compound refractive lenses.

- An imaging throw from target that provides at least $10 \mathrm{~m}$ between the target area and the detector.

\section{LCLS Trends}

Christine Sweeney (Los Alamos National Laboratory) presented an "out-brief" of the computational workshop on Automated Analysis and Control that was held during the LCLS Users meeting. This workshop explored options for developing generalized components that could be used in various scenarios to expedite automation. Advanced automation concepts would apply statistically-based methods of adaptive sampling, streaming variational inference, and emulation to maximize the scientific gains during an experiment. An ongoing difficulty of computational automation has been that while primarily novel experiments receive beam time allocations at highly competitive facilities such as LCLS, they tend to be more difficult to automate. Developing generalized computational building blocks is proposed to advance automation efforts for these novel experiments.

Andy Aquila (SLAC) described the Tender X-ray Instrument (TXI) that is under development as one of the new instruments in the Near Experimental Hall (NEH). TXI will allow overlap of X-ray beams from both the hard and soft undulators, which will enable unique experiments using independent $\mathrm{X}$-ray beams for both pumping and probing materials. This instrument will provide opportunities for non-linear and multi-dimensional X-ray spectroscopies, such as chemically-specific characterization of reactive flow (e.g., combustion, fuel sprays, aerosol chemistry). TXI is scheduled for commissioning during 2023 with standard user operations starting in October 2023. Arthur Campello (Cornell University) presented recent simulations to model crossing $X$-ray beams with orthogonal polarizations. These numerical simulations of the complex, non-linear beam interactions will be important for planning and interpreting TXI experiments.

\section{User Science at International XFEL HED Instruments}

\subsection{European XFEL}

Ulf Zastrau, head of the HED science instrument at the European XFEL [29], presented a status update on the instrument. HED operates on the SASE 2 beamline, one of three at European XFEL that can be run simultaneously. SASE 2 can achieve $5 \mathrm{keV}$ at $10^{12}$ photons $(0.8 \mathrm{~mJ})$ per pulse and up to $25 \mathrm{keV}$ at $10^{10}$ photons $40 \mu \mathrm{J}$ per pulse), and will be the first beamline to have self seeding installed, around mid-2020. The HED instrument is planned to have two high power lasers provided by the HiBEF consortium. A multi-100 TW Ti:Sapphire chirped pulse amplification (CPA) system from Amplitude Laser has been installed and is undergoing timing and focusing commissioning, with X-ray commissioning experiments planned for early 2020, and availability to users planned in late 2020. The $10 \mathrm{~Hz} 100 \mathrm{~J}$ shaped nanosecond DiPOLE laser has been completed in the UK, and it is expected to be available to users in late 2021. HED features two interaction chambers: IC1 and IC2. IC1 is a chamber for short and long pulse laser interaction with many options for X-ray spectroscopy. IC2 is optimized for X-ray diffraction and is removable on rails to support static and dynamic diamond anvil cells (DAC) experiments. Laser heating to the $0.5 \mathrm{eV}$ level will be supported as well as piezo-based dynamic compression with a strain rate 
of $10^{3} / \mathrm{s}$. The latter has a timescale well suited to the MHz burst pulse trains of the XFEL. The first community experiment on IC2 making use of the DACs was performed in early October.

The HED instrument was active in the European XFEL's Run 3. The first user experiment succeeded in measuring low-frequency structural dynamics in X-ray heated single crystal diamond, resolving inelastic $X$-ray scattering with $50 \mathrm{meV}$ resolution, similar to polycrystalline diamond results described at MEC [30]. Other user experiments in run 3 demonstrated the polarimetry capability, using a $45^{\circ}$ silicon polarizer, X-ray Raman spectroscopy of heated matter, and nanofocusing.

Zastrau presented two projects underway anticipating use of the multi-100 TW laser systems. First, a photon arrival monitor (PAM) system is being developed to synchronize between the X-ray and optical fs laser pulses. Building on techniques established at LCLS [31], the PAM uses both spectral and spatial encoding channels to record temporal jitter. Preliminary measurements show $230 \mathrm{fs}$ (RMS) jitter. The second project is to deploy SAXS diagnostic using two monochromatizing spherical HAPG crystals, with a gap for the main beam, to avoid direct line of sight and prevent broadband X-rays from reaching the detector. This scheme was demonstrated with a target consisting of $80 \mathrm{~nm}$ spheres. Currently, the HED instrument expects 15 five-shift weeks out of every half year run, intended to serve roughly $50 \%$ to regular proposals, slightly more than $25 \%$ to HiBEF priority, with the remaining shifts going to management reserve and in-house experiments.

\subsection{SACLA}

Nicholas Hartley (Helmsholtz Zentrum Dresden-Rossendorf) described experiments at the SPring-8 Angstrom Compact free electron LAser (SACLA) HED instrument-experimental hutch 5 (EH5) and gave an overview of current capabilities of this instrument [32]. Previous experiments from LCLS-MEC showed the formation of diamond from polystyrene under double-shock compression but not from single shocks [33]. The experiments at SACLA expanded on the polystyrene study by examining shock compression of polyethylene terephthalate (PET), which showed an expansion of the phase stability region for diamond formation.

In another experiment at SACLA, Hartley and coworkers used two-color pulses with variable delays (0-300 fs) to examine the kinetics of disordering in graphite. The disordering was shown to be highly anisotropic in which the in-plane reflections (00L) lose intensity very quickly while the out-of-plane reflections (HK0) persist for much longer ( $\sim 500 \mathrm{fs})$ [34].

\section{Recent Experiments at LCLS-MEC}

Users gave updates on each of the experiments that had been conducted since the previous workshop. First, Hiroshi Sawada (University of Nevada Reno) presented an experiment in short pulse laser-driven isochoric heating, in which spherical crystal imaging and X-ray Thomson scattering diagnostics were deployed. Tuned to register K-shell fluorescence, the X-ray imager indicated the degree of X-ray to optical laser overlap, showing that the pre-alignment procedure was imperfect. The experiment also showed that standard Thomson scattering measurements in the Von Hamos geometry, used successfully in long pulse shock experiments, suffers from excessive background signal in the case of relativistic intensity laser irradiation. These results further motivated the development by MEC scientists of transmission crystal spectrometers and more robust shielding designs in general for spectroscopy under high background conditions.

Thomas Kluge (Helmsholtz Zentrum Dresden-Rossendorf) presented an experiment in small angle X-ray scattering (SAXS) building on their previous published results from MEC [13]. The SAXS technique holds the potential to measure structural patterns in rapidly evolving plasmas with sub-micron scale lengths, such as Weibel instabilities. This experiment expanded on the MEC results by studying structured buried layers and tuning the XFEL to resonances. Asymmetry in the pattern reveals opacity and geometry, which in this case distinguished the expansion of a buried layer from that of the rear surface. 
Richard Briggs (Lawrence Livermore National Laboratory) reported on observations of liquid-liquid phase transitions under dynamic compression of selenium. He noted that shots had to be taken with two different LCLS photon energies-11 keV and $9 \mathrm{keV}$-in order to fill out the full Q-range needed to observe differences in liquid structure, and strongly advised creating a new standard configuration in which wide Q-range is prioritized, particularly in maintaining low $Q$ coverage as the higher photon energies of LCLS-II give access to higher Q. The experiment made use of a new open-source web-based data visualization tool called Cinema: Bandit [35], which Briggs and others endorsed as being helpful in facilitating real-time decisions about complex data sets.

Markus Schoelmerich (European XFEL) presented on the topic of $\mathrm{SiO}_{2}$ melting at ultrahigh pressures, which is relevant to geophysics, exoplanet interiors, and impact physics. Using the MEC long pulse laser, the team drove shocks up to $146 \mathrm{GPa}$ in quartz crystal (both c- and a-cut) and fused silica. They observed that, while both melt, the fused silica target remains amorphous on release, whereas the quartz targets showed formation of polycrystaline quartz at late times. The pair distribution function of the melted $\mathrm{SiO}_{2}$ showed a change in coordination at high pressure, but the calculations are difficult. Measurements of these states will benefit from the higher photon energy that will be made available in LCLS-II.

Thomas Preston (European XFEL) presented MEC investigations on the momentum dependence of plasmonic excitation in compressed $\mathrm{Al}$ [16] at around $1 \mathrm{Mbar}$ and in compressed $\mathrm{Cu}$ up to $5 \mathrm{Mbar}$, with results coming from two different MEC experimental runs. These measurements used the spectrometer that was in the LCLS front end enclosure (FEE) and has since been removed.

Oliver Humphries (University of Oxford) presented on an experiment measuring electronic structure and valence electron rebinding in dense plasmas via resonant inelastic X-ray scattering. This experiment observed the K-alpha emission in $\mathrm{Ni}$ at and below the K-edge to examine the effects of heating and ionization in dense plasmas.

Philip Hart (SLAC) discussed recent detector development efforts. The CSPAD quads that have been used at MEC since 2013 are being replaced with ePix10k quads. These detectors permit measurement of $10 \mathrm{k}$ photons per pixel, and they have a $1 \mathrm{~mm}$ silicon sensor for better efficiency with the higher energy X-rays from LCLS-II. ePIX100 detectors will still be used for spectroscopy, and ptychography will be performed with Jungfrau or ePIX10k detectors. Testing has also been performed recently with nanosecond framing cameras, including the Icarus ultrafast X-ray imaging detector [36]. Efforts to alleviate the longstanding issues with high energy laser shots generating detrimental EMP are continuing with better triggering, grounding, and shielding in the ePIX10k detectors.

Additional information may be obtained at the workshop website: https:/ conf.slac.stanford. edu/hpl-2019/.

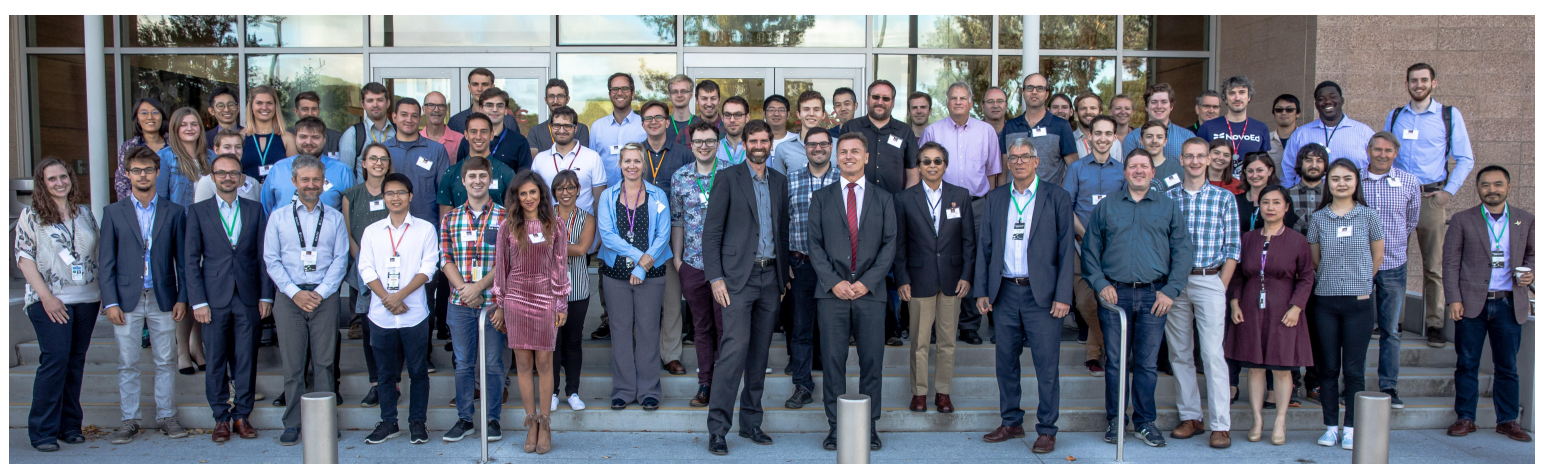


Funding: Workshop funding was provided by the DOE Office of Science-Fusion Energy Sciences under contract FWP 100189. We are grateful for the support of the corporate workshop sponsors: Prism Computational Sciences, Inc., Light Conversion, Phasics Corp., General Atomics, and Lawrence Livermore National Laboratory. Additional support was provided by SLAC National Accelerator Laboratory, Stanford University, and Los Alamos National Laboratory.

Acknowledgments: We also gratefully acknowledge conference planning and administrative support from Nitssia Harrison and Carol Reynolds (SLAC National Accelerator Laboratory). Photos by Dawn Harmer.

Conflicts of Interest: The authors declare no conflicts of interest.

\section{References}

1. Falcone, R.; Glenzer, S.; Hau-Riege, S. User Workshop on High-Power Lasers at the Linac Coherent Light Source. Synchrotron Radiat. News 2014, 27, 56-58. [CrossRef]

2. Heimann, P.; Glenzer, S. Second User Workshop on High-Power Lasers at the Linac Coherent Light Source. Synchrotron Radiat. News 2015, 28, 54-56. [CrossRef]

3. Bolme, C.; Glenzer, S.; Fry, A. Third User Workshop on High-Power Lasers at the Linac Coherent Light Source. Synchrotron Radiat. News 2016, 29, 14-17. [CrossRef]

4. Bolme, C.; Mackinnon, A.; Glenzer, S. Fourth User Workshop on High-Power Lasers at the Linac Coherent Light Source. Synchrotron Radiat. News 2017, 30, 58-62. [CrossRef]

5. Bolme, C.; Galtier, E.; Glenzer, S. Fifth user workshop on high-power lasers at the linac coherent light source. Powder Diffr. 2018, 33, 259-263. [CrossRef]

6. Bolme, C.; Dyer, G.; Glenzer, S. Sixth user workshop on high-power lasers at the linac coherent light source. Powder Diffr. 2019, 34, 79-84. [CrossRef]

7. Brown, S.B.; Gleason, A.E.; Galtier, E.; Higginbotham, A.; Arnold, B.; Fry, A.; Granados, E.; Hashim, A.; Schroer, C.G.; Schropp, A.; et al. Direct imaging of ultrafast lattice dynamics. Sci. Adv. 2019, 5, eaau8044. [CrossRef] [PubMed]

8. Coleman, A.; Gorman, M.; Briggs, R.; McWilliams, R.; McGonegle, D.; Bolme, C.; Gleason, A.; Fratanduono, D.; Smith, R.; Galtier, E.; et al. Identification of Phase Transitions and Metastability in Dynamically Compressed Antimony Using Ultrafast X-Ray Diffraction. Phys. Rev. Lett. 2019, 122, 255704. [CrossRef] [PubMed]

9. Gorman, M.G.; Coleman, A.L.; Briggs, R.; McWilliams, R.S.; McGonegle, D.; Bolme, C.A.; Gleason, A.E.; Galtier, E.; Lee, H.J.; Granados, E.; et al. Femtosecond diffraction studies of solid and liquid phase changes in shock-compressed bismuth. Sci. Rep. 2018, 8, 16927. [CrossRef] [PubMed]

10. Gorman, M.G.; Coleman, A.L.; Briggs, R.; McWilliams, R.S.; Hermann, A.; McGonegle, D.; Bolme, C.A.; Gleason, A.E.; Galtier, E.; Lee, H.J.; et al. Recovery of metastable dense Bi synthesized by shock compression. Appl. Phys. Lett. 2019, 114, 120601. [CrossRef]

11. Hartley, N.J.; Vorberger, J.; Döppner, T.; Cowan, T.E.; Falcone, R.W.; Fletcher, L.B.; Frydrych, S.; Galtier, E.; Gamboa, E.J.; Gericke, D.O.; et al. Liquid Structure of Shock-Compressed Hydrocarbons at Megabar Pressures. Phys. Rev. Lett. 2018, 121, 793-796. [CrossRef] [PubMed]

12. Helfrich, J.; Vorberger, J.; Frydrych, S.; Schaumann, G.; Ravasio, A.; Gauthier, M.; Fletcher, L.B.; Nagler, B.; Barbrel, B.; Bachmann, B.; et al. Investigation of the temperature in dense carbon near the solid-liquid phase transition between $100 \mathrm{GPa}$ and 200 GPa with spectrally resolved X-ray scattering. High Energy Density Phys. 2019, 32, 56-62. [CrossRef]

13. Kluge, T.; Rödel, M.; Metzkes-Ng, J.; Pelka, A.; Garcia, A.L.; Prencipe, I.; Rehwald, M.; Nakatsutsumi, M.; McBride, E.E.; Schönherr, T.; et al. Observation of Ultrafast Solid-Density Plasma Dynamics Using Femtosecond X-Ray Pulses from a Free-Electron Laser. Phys. Rev. X 2018, 8, 031068. [CrossRef]

14. Kraus, D.; Bachmann, B.; Barbrel, B.; Falcone, R.W.; Fletcher, L.B.; Frydrych, S.; Gamboa, E.J.; Gauthier, M.; Gericke, D.O.; Glenzer, S.H.; et al. Characterizing the ionization potential depression in dense carbon plasmas with high-precision spectrally resolved x-ray scattering. Plasma Phys. Control. Fusion 2018, 61, 014015-014017. [CrossRef]

15. McBride, E.E.; Krygier, A.; Ehnes, A.; Galtier, E.; Harmand, M.; Konopkova, Z.; Lee, H.J.; Liermann, H.P.; Nagler, B.; Pelka, A.; et al. Phase transition lowering in dynamically compressed silicon. Nat. Phys. 2018, 27, 291. [CrossRef] 
16. Preston, T.R.; Appel, K.; Brambrink, E.; Chen, B.; Fletcher, L.B.; Fortmann-Grote, C.; Glenzer, S.H.; Granados, E.; Göde, S.; Konopkova, Z.; et al. Measurements of the momentum-dependence of plasmonic excitations in matter around $1 \mathrm{Mbar}$ using an X-ray free electron laser. Appl. Phys. Lett. 2019, 114, 014101-014106. [CrossRef]

17. Tracy, S.J.; Smith, R.F.; Wicks, J.K.; Fratanduono, D.E.; Gleason, A.E.; Bolme, C.A.; Prakapenka, V.B.; Speziale, S.; Appel, K.; Fernandez-Pañella, A.; et al. In situ observation of a phase transition in silicon carbide under shock compression using pulsed x-ray diffraction. Phys. Rev. B 2019, 99, 214106. [CrossRef]

18. Witte, B.; Röpke, G.; Neumayer, P.; French, M.; Sperling, P.; Recoules, V.; Glenzer, S.; Redmer, R. Comment on "Isochoric, isobaric, and ultrafast conductivities of aluminum, lithium, and carbon in the warm dense matter regime". Phys. Rev. E 2019, 99, 047201. [CrossRef]

19. The LCLS-II: A High Power Upgrade to the LCLS Proc. 9th International Particle Accelerator Conference (IPAC'18), Vancouver, BC, Canada, April 29-May 4, 2018; JACoW Publishing: Geneva, Switzerland, 2018; Volume 9.

20. Marcus, G.; Anton, J.W.J.; Assoufid, L.; Decker, F.J.; Gassner, G.L.; Goetze, K.; Halavanau, A.; Hastings, J.B.; Huang, Z.; Jansma, W.G.; et al. Cavity-Based Free-Electron Laser Research and Development: A Joint Argonne National Laboratory and SLAC National Laboratory Collaboration; Number 39 in Free Electron Laser Conference; JACoW Publishing: Geneva, Switzerland, 2019; pp. 282-287. [CrossRef]

21. Dunne, M. LCLS Strategic Facility Development Plan; Report; SLAC National Accelerator Laboratory: Menlo Park, CA, USA, 2019.

22. National Academies of Sciences, Engineering, and Medicine. Opportunities in Intense Ultrafast Lasers: Reaching for the Brightest Light; National Academies Press: Washington, DC, USA, 2018. [CrossRef]

23. Falcone, R.; Albert, F.; Beg, F.; Glenzer, S.; Ditmire, T.; Spinka, T.; Zuegel, J. Workshop Report: Brightest Light Initiative (March 27-29 2019, OSA Headquarters, Washington, D.C.). arXiv 2020, arXiv:2002.09712.

24. Liang, T.T.; Bauer, J.M.; Liu, J.C.; Rokni, S.H. Radiation Protection Around High-intensity Laser Interactions with Solid Targets. Health Phys. 2018, 115, 687-697. [CrossRef]

25. Boland, M.; Tanaka, H.; Button, D.; Dowd, R.; Schaa, V.R.; Tan, E. (Eds.) Four X-ray Pulses within 10 ns at LCLS; JACoW Publishing: Geneva, Switzerland; Melbourne, Australia, 2019.

26. Blaj, G.; Dragone, A.; Kenney, C.J.; Abu-Nimeh, F.; Caragiulo, P.; Doering, D.; Kwiatkowski, M.; Markovic, B.; Pines, J.; Weaver, M.; et al. Performance of ePix10K, a high dynamic range, gain auto-ranging pixel detector for FELs. AIP Conf. Proc. 2019, 2054, 060062. [CrossRef]

27. Cunningham, E.; Galtier, E.; Dyer, G.; Robinson, J.; Fry, A. Pulse contrast enhancement via non-collinear sum-frequency generation with the signal and idler of an optical parametric amplifier. Appl. Phys. Lett. 2019, 114, 221106. [CrossRef]

28. Klisnick, A.; Menoni, C.S. (Eds.) Ultrafast Laser-Matter Interaction with Nanostructured Targets; SPIE: Bellingham, WA, USA, 2019.

29. Nakatsutsumi, M.; Appel, K.; Priebe, G.; Thorpe, I.; Pelka, A.; Muller, B.; Tschentscher, T. Technical Design Report: Scientific Instrument High Energy Density Physics (HED); Technical Report XFEL.EU TR-2014-001; DESY: Hamburg, Germany, 2014. [CrossRef]

30. McBride, E.E.; White, T.G.; Descamps, A.; Fletcher, L.B.; Appel, K.; Condamine, F.P.; Curry, C.B.; Dallari, F.; Funk, S.; Galtier, E.; et al. Setup for meV-resolution inelastic X-ray scattering measurements and X-ray diffraction at the Matter in Extreme Conditions endstation at the Linac Coherent Light Source. Rev. Sci. Instrum. 2018, 89, 10F104. [CrossRef] [PubMed]

31. Krupin, O.; Trigo, M.; Schlotter, W.F.; Beye, M.; Sorgenfrei, F.; Turner, J.J.; Reis, D.A.; Gerken, N.; Lee, S.; Lee, W.S.; et al. Temporal cross-correlation of $\mathrm{x}$-ray free electron and optical lasers using soft x-ray pulse induced transient reflectivity. Opt. Express 2012, 20, 11396-11406. [CrossRef] [PubMed]

32. Yabuuchi, T.; Kon, A.; Inubushi, Y.; Togahi, T.; Sueda, K.; Itoga, T.; Nakajima, K.; Habara, H.; Kodama, R.; Tomizawa, H.; et al. An experimental platform using high-power, high-intensity optical lasers with the hard $\mathrm{X}$-ray free-electron laser at SACLAThis article will form part of a virtual special issue on X-ray free-electron lasers. J. Synchrotron Radiat. 2019, 26, 585-594. [CrossRef]

33. Kraus, D.; Vorberger, J.; Pak, A.; Hartley, N.J.; Fletcher, L.B.; Frydrych, S.; Galtier, E.; Gamboa, E.J.; Gericke, D.O.; Glenzer, S.H.; et al. Formation of diamonds in laser-compressed hydrocarbons at planetary interior conditions. Nat. Astron. 2017, 1, 606-611. [CrossRef]

34. Hartley, N.; Grenzer, J.; Lu, W.; Huang, L.; Inubushi, Y.; Kamimura, N.; Katagiri, K.; Kodama, R.; Kon, A.; Lipp, V.; et al. Ultrafast anisotropic disordering in graphite driven by intense hard X-ray pulses. High Energy Density Phys. 2019, 32, 63-69. [CrossRef] 
35. Orban, D.; Banesh, D.; Tauxe, C.; Biwer, C.; Biswas, A.; Saavedra, R.; Sweeney, C.; Sandberg, R.; Bolme, C.; Ahrens, J.; et al. Cinema:Bandit: A visualization application for beamline science demonstrated on XFEL shock physics experiments. J. Synchrotron Radiat. 2020, 27, 1-10. [CrossRef]

36. Hart, P.A.; Carpenter, A.; Claus, L.; Damiani, D.; Dayton, M.; Decker, F.J.; Gleason, A.; Heimann, P.; Hurd, E.; McBride, E.; et al. First X-ray test of the Icarus nanosecond-gated camera. In X-Ray Free-Electron Lasers: Advances in Source Development and Instrumentation V; Tschentscher, T., Patthey, L., Tiedtke, K., Zangrando, M., Eds.; International Society for Optics and Photonics, SPIE: Bellingham, WA, USA, 2019; Volume 11038, pp. 31-39. [CrossRef]

(C) 2020 by the authors. Licensee MDPI, Basel, Switzerland. This article is an open access article distributed under the terms and conditions of the Creative Commons Attribution (CC BY) license (http://creativecommons.org/licenses/by/4.0/). 